\title{
Temperature compensated magnetic field sensor based on Love waves
}

\author{
Harshad Mishra ${ }^{1}$, Jérémy Streque ${ }^{1}$, Michel Hehn ${ }^{1}$, Prince Mengue ${ }^{1}$, Hamid \\ M'Jahed $^{1}$, Daniel Lacour ${ }^{1}$, Karine Dumesnil ${ }^{1}$, Sébastien Petit-Watelot ${ }^{1}$, Sergei \\ Zhgoon ${ }^{2}$, Vincent Polewczyk ${ }^{1}$, Aurélien Mazzamurro ${ }^{3}$, Abdelkrim Talbi ${ }^{3}$, Sami \\ Hage-Ali ${ }^{1}$, Omar Elmazria ${ }^{1}$ \\ ${ }^{1}$ Institut Jean Lamour, Université de Lorraine - CNRS, UMR 7198, F-54000, Nancy, France \\ ${ }^{2}$ National Research University Moscow Power Engineering Institute, 14 Krasnokazarmennaja \\ 111250 Moscow, Russia \\ ${ }^{3}$ Univ. Lille, CNRS, Centrale Lille, ISEN, Univ. Valenciennes, UMR - 8520 - IEMN, LIA \\ LICS/LEMAC, F-59000 Lille, France \\ E-mail : harshad.vr1@gmail.com; omar.elmazria@univ-lorraine.fr
}

\begin{abstract}
A temperature compensated magnetic field sensor based on the combination of CoFeB ferromagnetic thin films and Quartz/ZnO Love waveguide platform is developed and optimized. The Love wave is a shear horizontal guided wave and therefore provides an optimal interaction with magnetisation in the magneto-elastic thin film resulting in higher acoustic wave magneto-elastic coupling compared to the conventional Rayleigh wave based devices. ST-cut Quartz was chosen as substrate, $\mathrm{ZnO}$ as insulating layer for Love wave generation and temperature coefficient of frequency (TCF) compensation and $\mathrm{CoFeB}$ as the magnetostrictive layer sensitive to magnetic field. Experimental results show a magneto-acoustic sensitivity of 15.53 MHz/T with almost zero TCF.
\end{abstract}

Keywords: SAW devices, resonators, piezoelectric, Love waves, magnetic field sensor, temperature compensated

\section{Introduction}

Huge strides in miniaturization of devices have led to revolutions in the telecommunication industry. Surface acoustic wave devices have emerged as a pioneer in the areas of Radio-Frequency (RF) filters and resonators in applications related to satellite receivers, remote control systems, mobile phones and keyless entry systems. SAW technology is experiencing a new phase of development to meet the requirements of $5 \mathrm{G}$. These developments concern especially the micro-electromechanical-systems (MEMS) that exploit piezoelectric on insulator (POI) structures [1]. SAW devices have evolved as cutting-edge structures, designed and created using the technologies of micro-fabrication and inherent physical properties of the constituent materials.

Surface acoustic wave devices are based on the generation, transmission and reception of mechanical surface waves on an elastic medium known as a substrate. Due to their sensitivity to different physical and chemical stimuli, SAW devices offer excellent capabilities for use as sensors [2-4]. Not only are they miniaturized in size, but are also very robust. Due to the use of a piezoelectric substrate, these devices are completely passive and operate without any external power source. As per need, they may also be developed into packageless devices operating wirelessly $[5,6]$. 
It has also been reported that fabricating the SAW device with a magnetostrictive material allows the sensing of magnetic field. Due to the magnetostrictive strains and changes in elastic constants, an external magnetic field yields changes in the effective acoustic wave velocity and thus in the resonant frequency of the SAW device [7-9]. Thus there is a lot of publications on the development of Magnetic SAW (MSAW) sensors that use either a simple magnetostrictive material or an alloy combining different materials and with enhanced magnetoelastic properties [7,8,10,11, 12,13].

Several studies have been made to obtain an optimal MSAW device. Kadota et al. [6], El Hosni et al. [7] and Webb et al. [14] have considered $\mathrm{Ni}$ as the sensitive material, while on the other hand, Yamaguchi et al. [9] examined amorphous $\mathrm{TbFe}_{2}$ for their studies. Zhou et al. [15] also considered $\left[\mathrm{TbCo}_{2}-\mathrm{FeCo}\right]$ multilayer stacks as the sensitive material. Most of these studies have appraised a delay line geometry for their SAW device [7, 11, 14,15]. These studies primarily focus on the MSAW responses of Rayleigh waves or ShearHorizontal waves in either $\mathrm{LiNbO}_{3}$ or Quartz substrates. Later, the focus has been shifting towards the exploration of MSAW responses of Love waves. These Love waves are guided Shear-horizontal waves, generated in a layered acoustic wave structure with a guiding layer that ensures a confinement of the acoustic energy near the sensing surface, thus increasing the sensitivity.

Kittmann et al. [16] have shown that Love waves sensors exhibit a much higher sensitivity to magnetic fields and therefore are very apt for designing high-sensitivity-low-field magnetic sensors. Considering the displacements in a ShearHorizontal wave (inplane transverse) and the orientation of the magnetic anisotropy (also inplane), it is imperative that the interaction between the magnetic moments and the acoustic wave is stronger when compared to that in the case of Rayleigh waves in which the primary displacement is out-ofplane.

The objective of this paper is to demonstrate a SAW device for magnetic field detection with self-temperature effect compensation, i.e. with zero Temperature Coefficient of Frequency (TCF). A multi-layered SAW resonator [CoFeB/ZnO/Quartz (on a $90^{\circ}$ rotated ST-cut substrate)] is investigated experimentally. The considered cut of Quartz exhibits a positive temperature coefficient of frequency (TCF) and is compensated by the use of $\mathrm{ZnO}$ with its negative TCF. The role of the $\mathrm{ZnO}$ film is thus double: Love wave propagation, and TCF compensation. In this paper we detail the design of such a sensor, then provide the experimental data regarding the magnetization properties of the device and the radio frequency (RF) measurements under the influence of temperature and magnetic field.

\section{Device design and fabrication}

Synchronous single-port SAW resonators were considered in this study for their compatibility with wireless measurements. Using photolithography and lift-off processes, resonators with a wavelength $(\lambda)$ of $10 \mu \mathrm{m}$ were fabricated, with $100 \mathrm{~nm}$ thick Aluminium electrodes. The synchronous resonator consists of 100 electrode pairs or interdigital transducers (IDTs) and two sets of 200 electrode reflectors on each end of the device. The aperture was fixed to $40 \lambda$.

The fundamental resonance frequencies of the test devices thus ranged between 400 and $500 \mathrm{MHz}$ and the aim here is to address the industrial, scientific and medical (ISM) band at $433 \mathrm{MHz}$. The devices were oriented such that the generated acoustic waves propagate perpendicular to the $\mathrm{X}$ crystallographic direction of the ST-cut quartz substrate. This

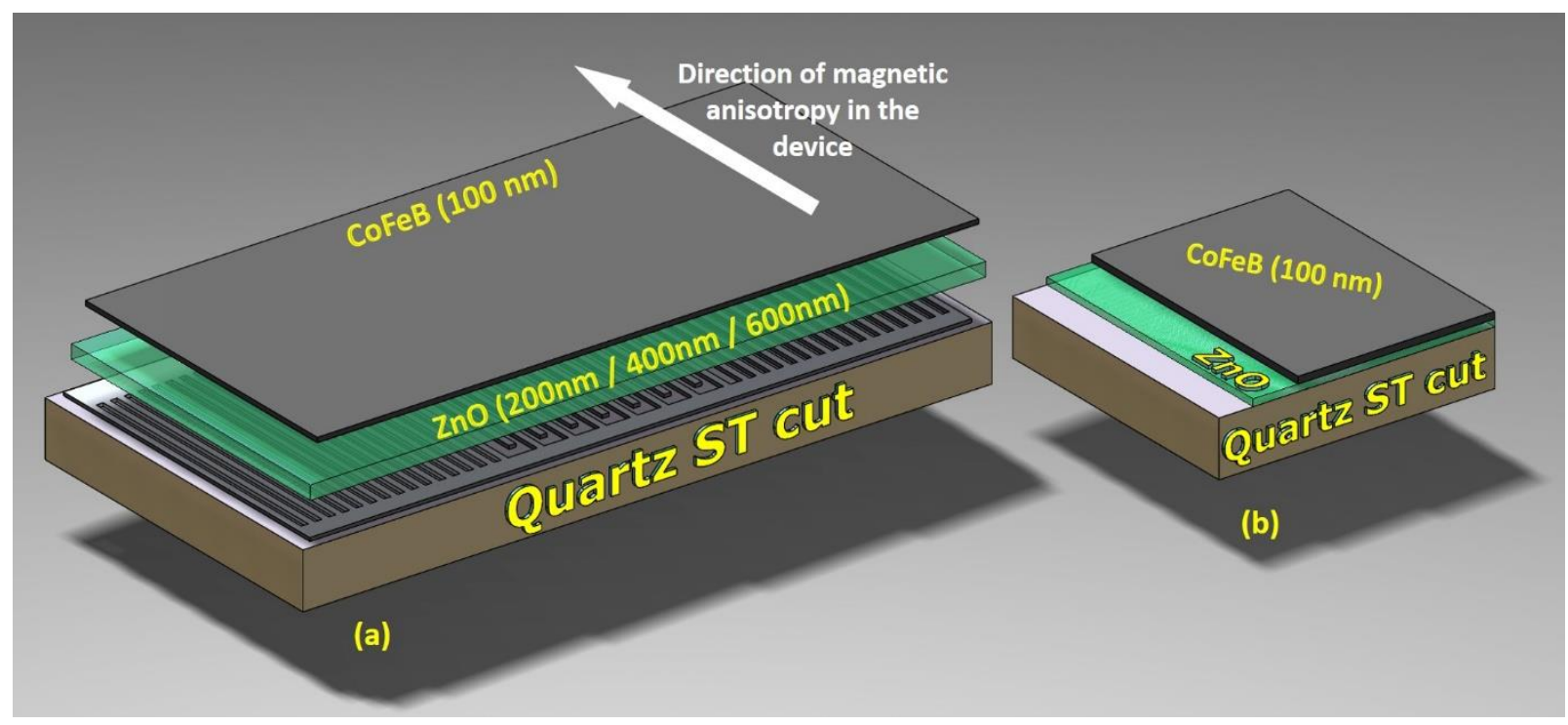

Figure 1: Schematic of the devices fabricated for the study: (a) multi-layered SAW resonator (b) multi-layered full film structure (without electrodes) for magnetometry tests. 


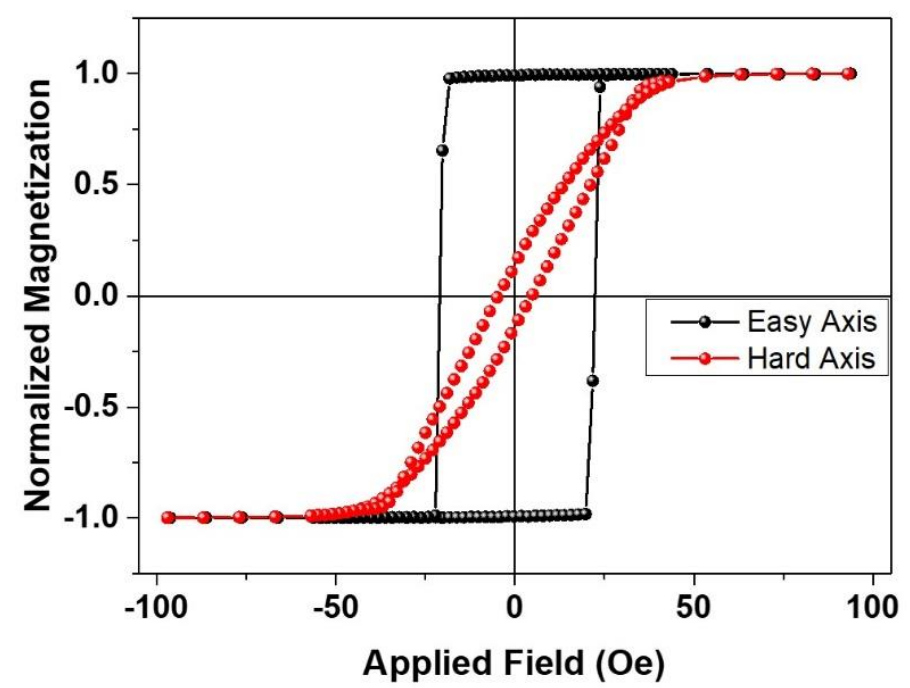

Figure 2: In-plane room temperature magnetisation measurements of the deposited magnetic layers (100nm of CoFeB over 200nm of ZnO on ST cut Quartz substrate).

enabled the excitation of the shear horizontal wave (which becomes a Love wave with addition of lower velocity layers).

A series of devices was then covered with $\mathrm{ZnO}$ layers of various thicknesses, obtained by $\mathrm{RF}$ sputtering of a 4-inch oxygen rich target at $150 \mathrm{~W}$ with $3 \times 10^{-3} \mathrm{mbar}$ and $8 \mathrm{~cm}^{3} / \mathrm{min}$ flow rate of both $\mathrm{Ar}$ and $\mathrm{O}_{2}$. The optimal $\mathrm{ZnO}$ thickness to obtain a fully temperature-compensated structure (without the magnetic layer) was consistently determined at 510nm. Subsequently, a 100nm thick CoFeB layer was sputtered by DC sputtering of a 2 -inch target at $300 \mathrm{~W}$ and $5 \times 10^{-3} \mathrm{mbar} \mathrm{Ar}$ over the $\mathrm{ZnO}$ layer to impart the capability of magnetic field sensing to the device. CoFeB was chosen as the sensitive layer for its low intrinsic magnetic anisotropy. The TCF was observed to drop towards negative values, thereby leading to a reduction of the optimal $\mathrm{ZnO}$ thickness for TCF compensation.

As a next step three different $\mathrm{ZnO}$ thicknesses were deposited on the blank devices, which are 200nm, 400nm, and 600nm (Samples D1, D2 and D3 respectively) and a CoFeB layer was deposited on each of the devices. The $\mathrm{CoFeB}$ layer thickness was kept constant at $100 \mathrm{~nm}$ on all the devices, to prevent an out-of-plane component of magnetization. Figure (a) shows a schematic explanation of the fabricated devices. In order to study the magnetic properties of the $\mathrm{Co}_{40} \mathrm{Fe}_{40} \mathrm{~B}_{20}$ film, a $100 \mathrm{~nm}$ thick CoFeB layer was deposited on a $200 \mathrm{~nm}$ thick $\mathrm{ZnO}$ layer previously deposited on a $4 \times 4 \mathrm{~mm}^{2}$ ST-cut quartz substrate (Sample V1). ZnO being a non-magnetic layer, has no effect on the magnetic characteristics. Therefore, only one $\mathrm{ZnO}$ thickness, i.e. $200 \mathrm{~nm}$, was used for this part of the study. Figure 1(b) shows the schematic explanation of the fabricated sample.

Magnetic characterization of the sample V1 has been conducted using a Vibrating Sample Magnetometer (VSM), with the field applied in-plane. As usually observed, the deposition by sputtering of the magnetic layer led to the existence of an easy axis of magnetization, as shown in Figure 2 (black curve). The anisotropy in the $\mathrm{CoFeB}$ film is induced by the stray field of the magnetron under the $\mathrm{CoFeB}$ target. But other factors such as the shadowing effect during deposition, strain, etc. cannot be ruled out as well. The control of the deposition parameters allow to orient this anisotropy. Indeed, the multi-layered structure presents a square hysteretic reversal along one direction that is chosen to be aligned parallel to the electrodes in the final device. The magnetic response along the in-plane perpendicular direction (red curve) (normal to SAW propagation) is characteristic of a hard axis response with an anisotropy field close to $50 \mathrm{Oe}$.

\section{RF Characterisation and TCF compensation}

The $S_{11}$ scattering parameter of the fabricated devices were measured using a network analyser (Agilent PNA 5230A, Santa Clara, CA) and RF probe station (PM5 Suss MicroTech). The return loss spectrum obtained for the SAW device without any overlayers showed a resonance peak at $482 \mathrm{MHz}$ (refer Fig. 3(a)) and the TCF was measured at $+30 \mathrm{ppm} /{ }^{\circ} \mathrm{C}$ using the thermally controlled chuck of the RF probe station (refer Fig. 4 black curve for TCF). In order to understand the nature of the resonance, simulations were made using Comsol Multiphysics. Figure 3(a) also shows the total displacement at the resonance corresponds to a Surface-Skimming Bulk Wave (SSBW) mode with leakage into the substrate [17]. Theoretical study has shown that the positive TCF of the quartz could be compensated by the negative TCF of $\mathrm{ZnO}$ and a zero TCF could be achieved with around $500 \mathrm{~nm}$ of $\mathrm{ZnO}$ (the details of the calculations are not shown in the paper). Subsequently, a $\mathrm{ZnO}$ layer was deposited on the device to 

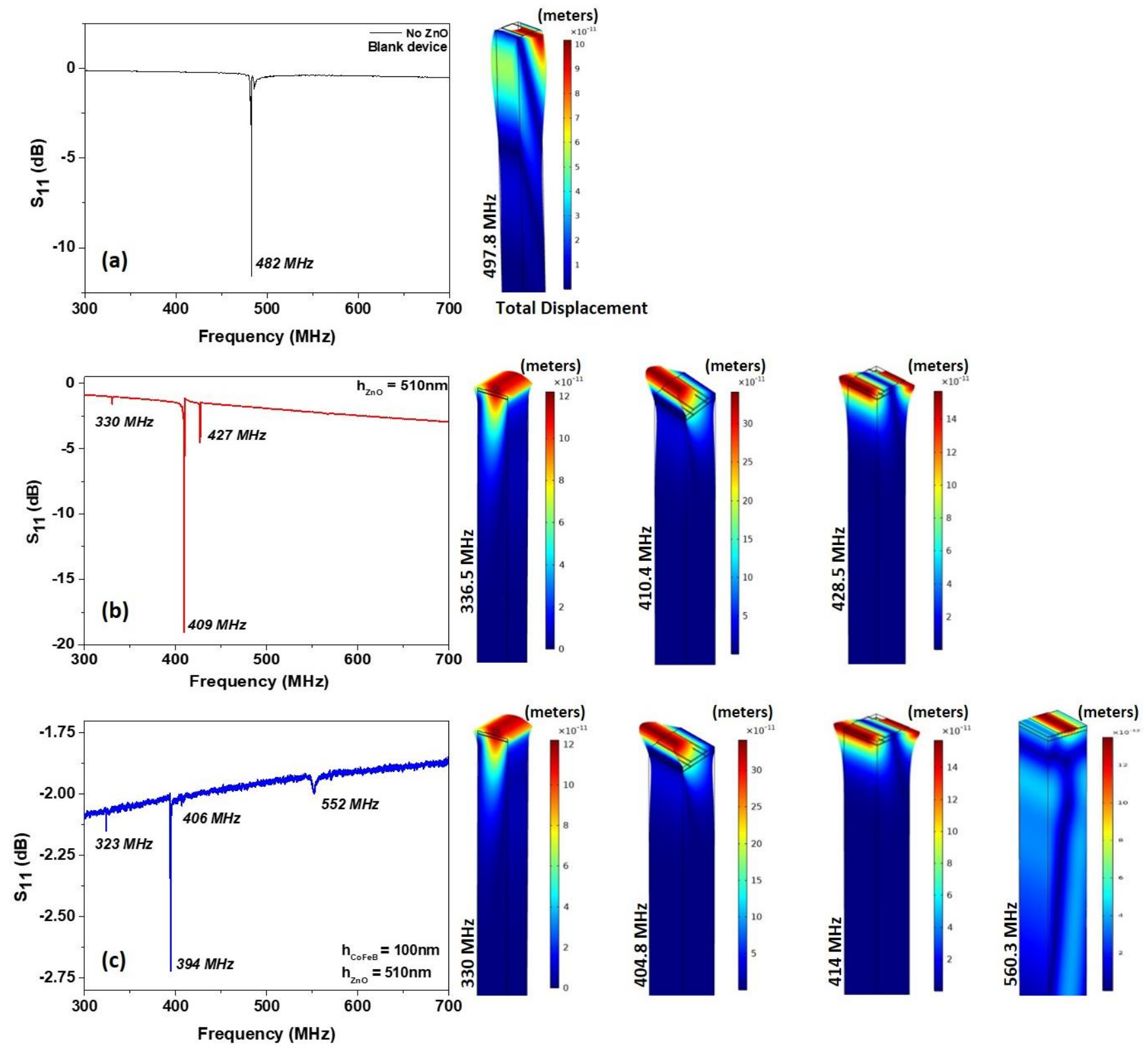

Figure 3: Experimental scattering parameters compared for (a) without $\mathrm{ZnO}$ on the blank device (b) with $510 \mathrm{~nm}$ of $\mathrm{ZnO}$ and (c) experimentally measured $S_{11}$ when $100 \mathrm{~nm}$ of CoFeB is added on top of the 510nm of ZnO. Total deformations for each resonance, obtained through simulations are presented beside each $S_{11}$.

compensate the positive TCF. The experimentally obtained $\mathrm{TCF}$ value was $-0.7 \mathrm{ppm} /{ }^{\circ} \mathrm{C}$ that is still remarkably close to zero. The $S_{11}$ spectra of this device is shown in Figure 3(b) and the TCF measurement in Figure 4, red curve. Comsol simulations (also in Figure 3(b)) reveal the presence of a Rayleigh wave mode at $330 \mathrm{MHz}$ followed by two ShearHorizontal modes at $409 \mathrm{MHz}$ and $427 \mathrm{MHz}$.

Following that, a $100 \mathrm{~nm}$ of $\mathrm{CoFeB}$ was deposited on top of the $\mathrm{ZnO}$. Figure 3(c) shows the return loss frequency behaviour after the addition of the $\mathrm{CoFeB}$ layer. The resonances seen before the additon of the $\mathrm{CoFeB}$ layer can also be seen after its additon. Comsol simulations peresented also reveal that the dip at $323 \mathrm{MHz}$ is a Rayleigh wave while those at $394 \mathrm{MHz}$ and $406 \mathrm{MHz}$ are Shear Horizontal in nature. A Bulk wave also appears $552 \mathrm{MHz}$ as confirmed by the simulations.

After the addition of the CoFeB layer on this device, it was observed that the TCF dropped to a negative value of -14.6 $\mathrm{ppm} /{ }^{\circ} \mathrm{C}$ (Figure 4 , green curve), indicating the need to reoptimize the $\mathrm{ZnO}$ thickness. However, thermal coefficients of elastic constants of $\mathrm{CoFeB}$ were not available in literature, thus making it impossible to theoretically determine the 


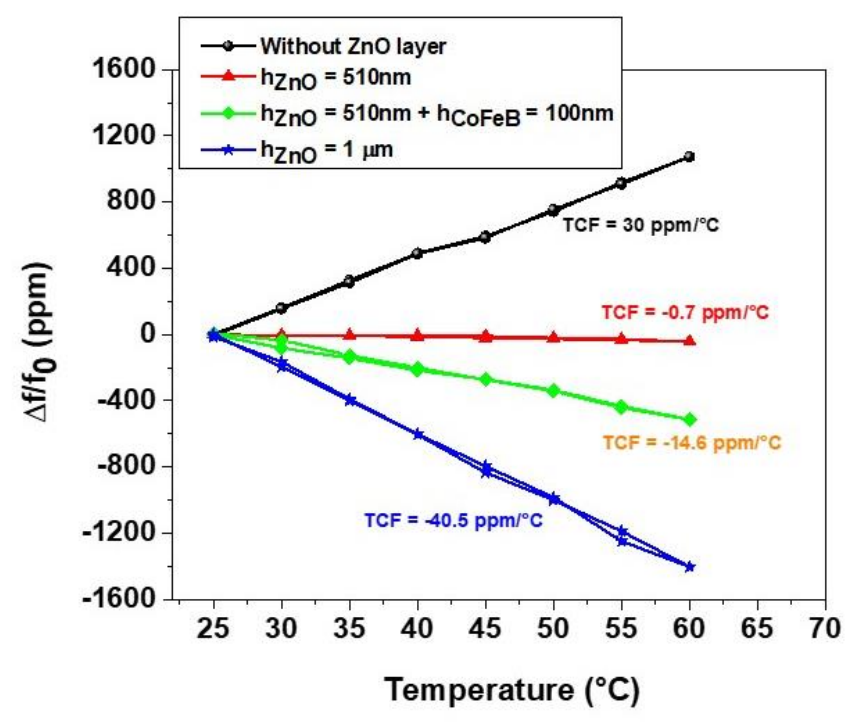

Figure 4: Dependence of frequency on temperature as measured on the different samples before the overlayers were deposited (black curve); with $1 \mu \mathrm{m}$ of $\mathrm{ZnO}$ on the

SAW device (blue curve); with 510nm of $\mathrm{ZnO}$ (red curve) and with overlayers of $100 \mathrm{~nm}$ of $\mathrm{CoFeB}$ and $510 \mathrm{~nm}$ of $\mathrm{ZnO}$ (green curve).

thickness of the different layers that allows a zero TCF device. Determination of the optimal thickness was therefore done experimentally. In this study, we keep the thickness of $\mathrm{CoFeB}$ constant and equal to $100 \mathrm{~nm}$ to avoid the modification of its magnetic properties and we vary the thickness of the $\mathrm{ZnO}$ layer in order to obtain a device with zero TCF. Thus, the devices D1, D2 and D3 with respective $\mathrm{ZnO}$ thicknesses of $200 \mathrm{~nm}, 400 \mathrm{~nm}$ and $600 \mathrm{~nm}$ were fabricated. A careful analysis of the resonance frequencies and a measurement of the TCFs helps us to determine that the frequencies observed in each case are $\mathrm{SH}$ waves observed earlier with $510 \mathrm{~nm}$ of $\mathrm{ZnO}$ (Figure 3(b)). Subsequently, with the addition of the $\mathrm{CoFeB}$ layers, a new resonance appears around $325 \mathrm{MHz}$ along with the $\mathrm{SH}$ resonances. By observing the incoherent TCF values for this peak (not shown in here) and also preliminary measurements of the frequency variation with magnetic field intensity, it can be deduced that this resonance is a Rayleigh or rather a quasi-Rayleigh wave.

Our experimental results show that the TCF of the CoFeB layer considered here is negative. Only $100 \mathrm{~nm}$ of $\mathrm{CoFeB}$ was enough to replace $310 \mathrm{~nm}$ of $\mathrm{ZnO}$. With just $100 \mathrm{~nm}$ of $\mathrm{CoFeB}$, deposited on $200 \mathrm{~nm}$ of $\mathrm{ZnO}$ it was possible to reach a TCF value of $1.77 \mathrm{ppm} /{ }^{\circ} \mathrm{C}$ (almost zero), whereas $510 \mathrm{~nm}$ of $\mathrm{ZnO}$ was necessary to obtain the same result. Alternately, a slightly thicker $\mathrm{ZnO}$ layer by around $10-20 \mathrm{~nm}$ is expected to get the device even closer to a zero TCF value. Finally, the multilayered structure with a $\mathrm{ZnO}$ thickness of $200 \mathrm{~nm}$ and $100 \mathrm{~nm}$ of $\mathrm{CoFeB}$ was selected for measurements under magnetic field. The Love wave observed in this device (Figure 5(a) red
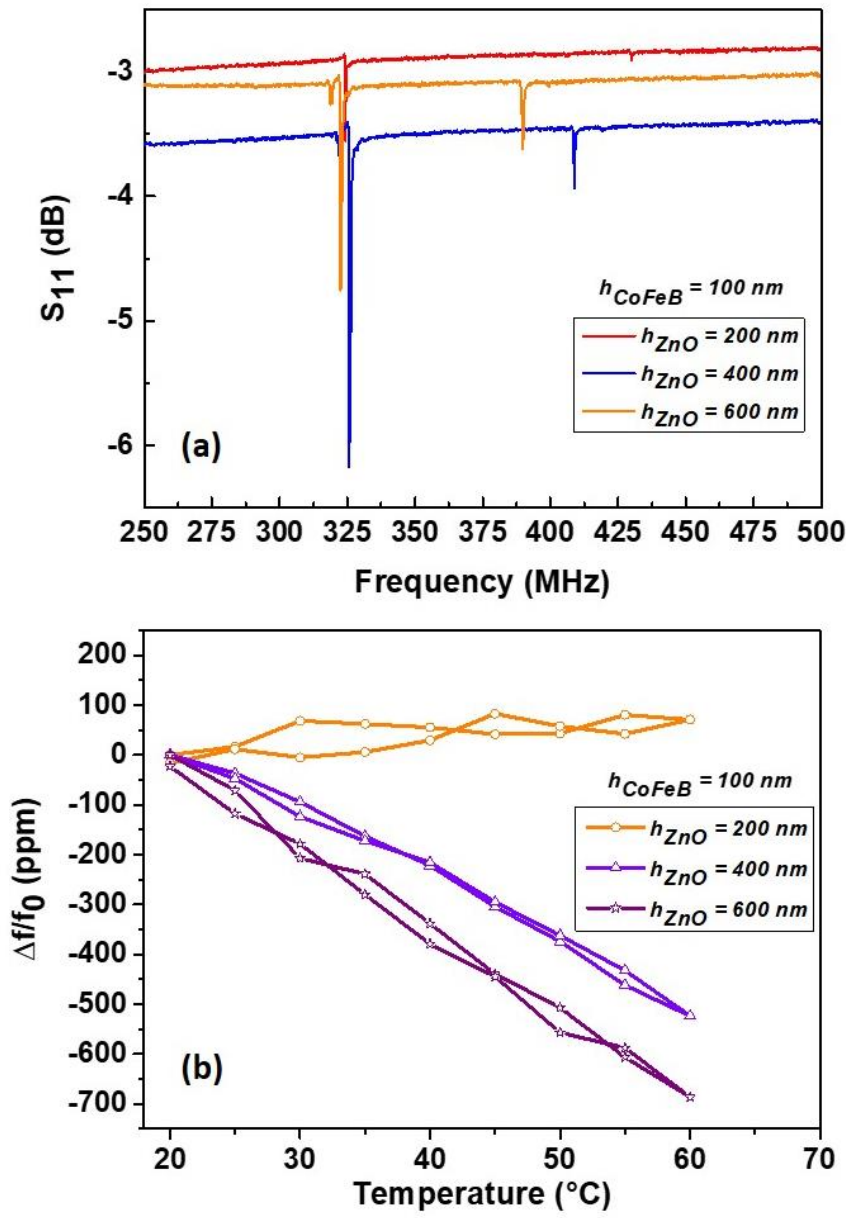

Figure 5: (a) Scattering parameters $\left(S_{11}\right)$ measured on the devices with overlayers of $100 \mathrm{~nm}$ of CoFeB and $200 \mathrm{~nm}$, $400 \mathrm{~nm}$ and $600 \mathrm{~nm}$ of $\mathrm{ZnO}$. The Love wave resonance is the small peak observed around the $400-425 \mathrm{MHz}$ range. (b) The relative variation of the Love wave frequency with respect to temperature for each device.

curve, shallow dip at $430 \mathrm{MHz}$ ) exhibits an operation frequency very close to the ISM band and a near-zero TCF.

Additionally, we observe that after the deposition of the CoFeB layer, the Love wave resonances become weaker (Figure 5(a)). Our understanding points to several different factors contributing to this. Indeed, deposition of high quality $\mathrm{ZnO}$ over the electrode area is a challenge due to the uneven surface created by the IDTs over the quartz substrate. Further, the presence of the metallic $\mathrm{CoFeB}$ so close to the electrodes leads to losses as well. However these losses can be eliminated with a better design and fabrication techniques in the future.

\section{Frequency variation under applied magnetic field}

The magneto-acoustic response of the Love wave peak in the temperature compensated $430 \mathrm{MHz}$ peak of the device (D1) is measured versus the direction and intensity of an 
applied magnetic field. The field is applied along two directions, parallel to the wave propagation direction of the device and perpendicular to it. As expected, due to the magnetic behaviour, the perpendicular orientation corresponds to the easy axis response while the parallel yields the hard axis response. The frequency variation obtained with respect to a varying magnetic field along the two directions are shown in Figure 6.
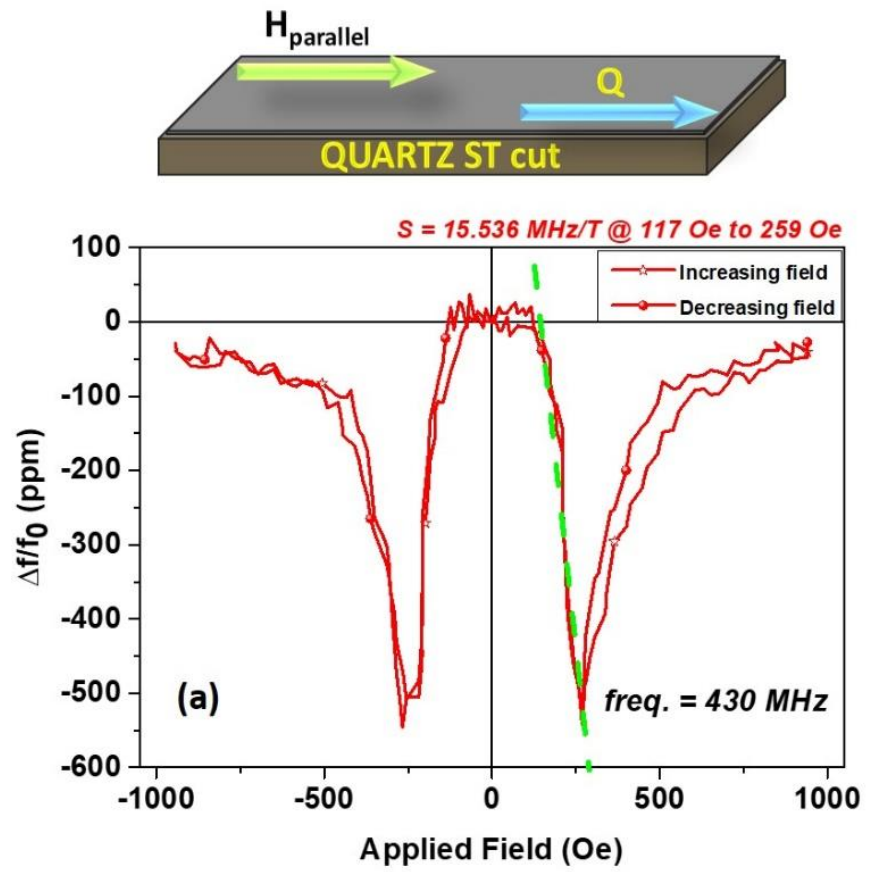

focused more on removing the zero magnetic field temperature effects and obtaining an equivalent sensitivity. Accordingly, to the purpose of this structure, the temperature effect observed for a $200 \mathrm{~nm}$ thick $\mathrm{ZnO}$ layer $\left(1.77 \mathrm{ppm} /{ }^{\circ} \mathrm{C}\right)$ only marginally interferes with the magnetic field measurement, which shows a sensitivity range of approximately $500 \mathrm{ppm}$. Thicknesses of $\mathrm{ZnO}$ and $\mathrm{CoFeB}$ could be optimised to obtain a zero TCF value and the
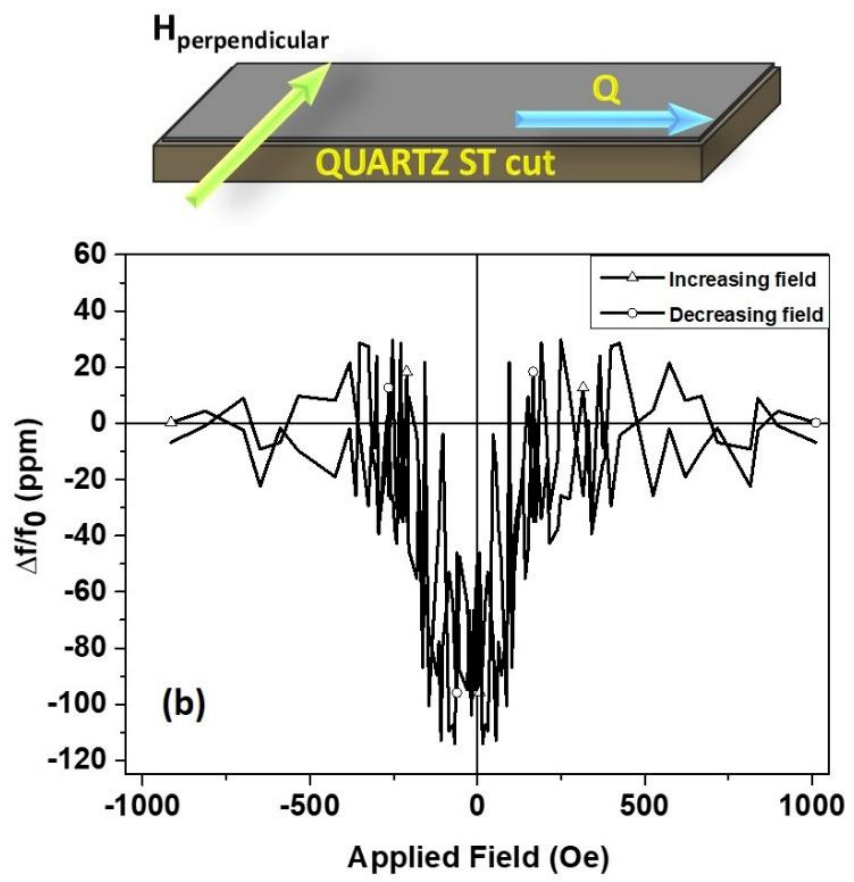

Figure 6: MSAW measurements on the temperature compensated device $\left(\mathrm{h}_{\mathrm{ZnO}}=200 \mathrm{~nm} ; \mathrm{h}_{\mathrm{CoFeB}}=100 \mathrm{~nm}\right)$ for the resonance peak at $430 \mathrm{MHz}$. The field is applied (a) along the hard axis i.e. parallel to wave propagation direction and (b) along the easy axis i.e. perpendicular to the wave propagation direction. The corresponding orientations are sketched above the figures with $\mathrm{Q}$ being the wave propagation direction and $\mathrm{H}$ the applied field. The sensitivity of $15.536 \mathrm{MHz} / \mathrm{T}$ is measured between 117 Oe and 259 Oe for the hard axis configuration shown in (a) and the linearity is highlighted by the green dotted line.

Since the other peaks are not temperature compensated, they are of little importance in this study and thus not discussed here. As can be seen, the sensitivity of the device is significantly higher with the field applied parallel to the wave propagation direction. The measurements show a monotonous, quasi-linear behaviour between 100 and 260 Oe, that can be exploited for magnetic field sensing applications. The response to magnetic fields perpendicular to the wave propagation direction being considerably lower, this confers this structure an acceptable directivity.

The measured sensitivity of the device is $15.536 \mathrm{MHz} / \mathrm{T}$ between 117 and 259 Oe. This is comparable to the sensitivity measured by Kittman et al. [15] who obtained around 44.24 $\mathrm{MHz} / \mathrm{T}$ (calculated from their experimental results) but much more sensitive compared to the results of Kadota et al. [7], Elhosni et al. [8] and Zhou et al. [14]. Although, Kittman et al. emphasised on obtaining a higher sensitivity, our device resonator design could be improved to enhance the quality of the sensor and thus enhance its sensitivity.

\section{Conclusions}

In this work we have presented a way to mitigate the effects of temperature on a SAW device used for magnetic field sensing. The positive TCF of a quartz-based device is compensated by a multi-layered structure made with $\mathrm{ZnO}$ and CoFeB. The TCF of the device has been measured at 1.77 $\mathrm{ppm} /{ }^{\circ} \mathrm{C}$, indicating that it is relatively free from the effects of temperature drifts during magnetic field measurement. Additionally, the device designed to operate in $433 \mathrm{MHz}$ ISM band has been shown to have a very good magnetic field sensitivity (around $15.5 \mathrm{MHz} / \mathrm{T}$ ). This sensitivity could be further improved by the optimisation of the resonator design and probably by increasing the operating frequency of the sensor. 


\section{Acknowledgements}

We would like to acknowledge the support from the French PIA project "Lorraine Université d'Excellence" (ANR-15IDEX-04-LUE, ANR JCJC SAWGOOD (ANR-18-CE420004-01), the Région Grand-Est and the European funds FEDER. Experiments were carried out on IJL Project TUBEDavm equipment funded by FEDER (EU), Region Grand Est, Métropole Grand Nancy. We also extend our sincere gratitude to the staff from the Competence Centre MINALOR for helpful discussions in fabrication.

\section{References}

[1] Mahon S., 2017 IEEE Trans. on Semiconductor Manuf., 30, 4

[2] Aubert T., Elmazria O., Assouar B., Bouvot L., and Oudich M., 2010 Appl. Phys. Lett. 96, 203503

[3] Talbi A., Sarry F., Elhakiki M., Le Brizoual L., Elmazria O., Nicolay P., and Alnot P., 2006, Sens. Actuators 128, 78

[4] Wohltjen H. and Dessy R., 1979, Anal. Chem. 51 (9) 1458-1475

[5] Bhattacharjee K., Shvetsov A., Zhgoon S., 2007 Proc. IEEE Freq. Control Symp. 135-140

[6] Krishnamurthy S., Bazuin B. J. and Atashbar M. Z., 2005 Proc. IEEE/EIT Conference, Lincoln, Nebraska

[7] Kadota M., Ito S., Ito Y., Hada T., and Okaguchi K., 2011, Jpn. J. Appl. Phys. 50, 07HD07

[8] M. Elhosni, O. Elmazria, S. Petit-Watelot, L. Bouvot, S. Zhgoon, A. Talbi, M. Hehn, K. Ait-Aissa, S. Hage-Ali, D. Lacour, F. Sarry, O. Boumatar ; 2016, Sensors and Actuators A, 240, 41-49

[9] Yamaguchi M., Hashimoto K. Y., Kogo H., and Naoe M., 1980 IEEE Trans. Magn. 16, 916

[10] Tiercelin N., Preobrazhensky V., Pernod P., and Ostaschenko A., 2008 Appl. Phys. Lett. 92, 062904

[11] Tiercelin N., Talbi A., Preobrazhensky V., Pernod P., Mortet V., Haenen K., and Soltani A., 2008 Appl. Phys. Lett. 93, 162902

[12] Polewczyk V., Dumesnil K., Lacour D., Moutaouekkil M., Mjahed H., Tiercelin N., Petit Watelot S., Mishra H., Dusch Y., Hage-Ali S., Elmazria O., Montaigne F., Talbi A., Bou Matar O., Hehn M., 2017, Phys. Rev. Appl. 8, 024001

[13] Mishra H., Hehn M., Lacour D., Elmazria O., Tiercelin N., Mjahed H., Dumesnil K., Petit Watelot S., Polewczyk V., Talbi A., Bou Matar O., Hage-Ali S., 2019, Smart Mater. Struct. 28, 12LT01

[14] Webb D. C., Forester D.W., Gangu1y A. K., and Vittoria C., 1979 IEEE Trans. Magn. 15, 1410

[15] Zhou H., Talbi A., Tiercelin N., and Bou Matar O., 2014 Appl. Phys. Lett. 104, 114101

[16] Kittmann A., Durdaut P., Zabel S., Reermann J., Schmalz J., Spetzler B., Meyners D., Sun N. X., McCord J., Gerken M., Schmidt G., Höft M., Knöchel R., Faupel F. and Quandt E., 2018 Scientific Reports 8278

[17] Caliendo C. and Hamidullah M., 2019, J. Phys. D: Appl. Phys. 52153001 